\title{
Highly efficient sources of single indistinguishable photons
}

\author{
Niels Gregersen \\ DTU Fotonik, Department of Photonics Engineering, \\ Technical University of Denmark \\ Kongens Lyngby, Denmark \\ ngre@fotonik.dtu.dk
}

\begin{abstract}
Solid-state sources capable of emitting single photons on demand are of great interest in quantum information applications. Ideally, such a source should emit exactly one photon into the collection optics per trigger, the emitted photons should be indistinguishable and the source should be electrically driven. Several design strategies addressing these requirements have been proposed. In the cavity-based source, light emission is controlled using resonant cavity quantum electrodynamics effects, whereas in the waveguide-based source, broadband electric field screening effects are employed to direct the light emission into the optical mode of interest. For all the strategies, accurate modeling and careful optical engineering is required to achieve high performance.
\end{abstract}

Keywords-Light-emitting diode; microcavity; photonic nanowire; single-photon source

\section{INTRODUCTION}

Within the emerging field of optical quantum information processing, the current challenge is to construct the basic building blocks for the quantum computing and communication systems. A key component is the singlephoton source (SPS) [1] capable of emitting single photons on demand. Ideally, the SPS must feature near-unity efficiency, where the efficiency is defined as the number of detected photons per trigger, the probability $g^{(2)}(\tau=0)$ of multi-photon emission events should be 0 and the emitted photons are required to be indistinguishable.

An optically or electrically triggered quantum light emitter, e.g. a nitrogen-vacancy center or a semiconductor quantum dot (QD), embedded in a solid-state semiconductor host material appears as an attractive platform for generating such single photons. However, for a QD in bulk material, the large index contrast at the semiconductor-air interface leads to a collection efficiency of only 1-2 \%, and efficient light extraction [2] thus poses a major challenge in SPS engineering.

Two parameters govern the efficiency of a SPS. [2] The first is the $\beta$ factor given by the ratio of spontaneous emission from the quantum emitter into the optical mode of interest. It is expressed as

$$
\beta=\frac{\Gamma_{M}}{\Gamma_{M}+\Gamma_{\text {Rad }}},
$$

where $\Gamma_{M}$ and $\Gamma_{\text {Rad }}$ are the spontaneous emission rates to the fundamental mode and to the radiation modes respectively.

The photon then escapes through a specifically designed loss channel to the collection optics with a probability described by the outcoupling coefficient $\gamma$. The total efficiency $\varepsilon$ is then simply given by $\varepsilon=\beta \gamma$.

\section{CAVITY-BASED SPS}

Inspection of (1) reveals two possible strategies for optimizing the spontaneous emission factor $\beta$. The first is to increase the spontaneous emission rate $\Gamma_{M}$ using cQED effects in the weak coupling regime. [3] In this approach the quantum emitter is placed in a microcavity of quality factor $Q$, mode volume $V$ and resonance wavelength $\lambda$.

If the emitter is located at the antinode of the cavity mode with its dipole moment aligned with the electric field and the emitter and the cavity are spectrally aligned, the spontaneous emission rate into the cavity mode is enhanced relative to the rate in a bulk medium by the Purcell factor [4] proportional to $Q / V$. A high-efficiency cavity-based SPS thus requires a large (a)

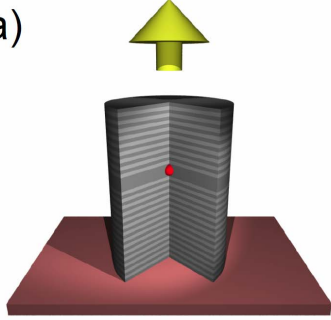

(c)
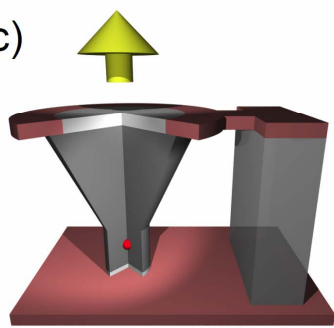

(b)

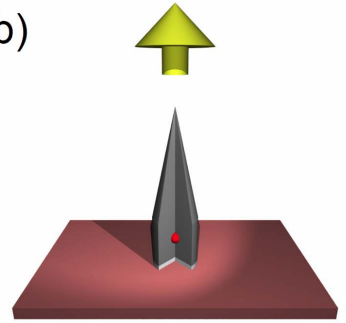

(d)

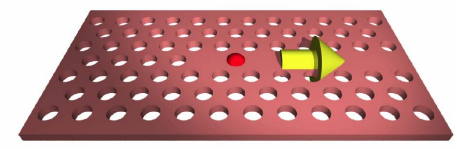

Fig. 1. Illustrations of different design strategies for single-photon sources are sketched. The (a) microcavity pillar, the photonic nanowire featuring a (b) regular (b) or an (c) inverted conical tapering and the (d) terminated W1 waveguide. The quantum emitter and the directivity of the light emission are illustrated by the red dot and the yellow arrow respectively. 
$Q$ factor combined with a low mode volume and the optical design challenge for the cavity-based SPS is to maximize the ratio $Q / V$ while maintaining a high outcoupling coefficient $\gamma$.

The most popular geometry for the cavity-based SPS is the micropillar cavity illustrated in Fig. 1(a). The micropillar is a vertical structure consisting of a cavity section with an embedded quantum emitter surrounded by distributed Bragg reflectors (DBRs). Optical mode confinement is ensured by the photonic bandgap of the DBRs along the vertical axis and by total internal reflection in the plane. In the ideal structure light escapes only along the vertical axis. A strongly asymmetrical structure is required to ensure that light is emitted predominantly in the forward direction. Very recently an efficiency of 0.79 was demonstrated [5] in a micropillar cavity fabricated using an in-situ technique. In the same work, a photon indistinguishability of 0.82 was measured for a slightly lower efficiency of 0.62 .

\section{WAVEGUIDE-BASED SPS}

A second possible approach for optimizing the $\beta$ factor as defined by (1) is to suppress the spontaneous emission contribution $\Gamma_{R a d}$ to radiation modes. This alternative strategy is employed in the photonic nanowire SPS designs illustrated in Fig. 1(b,c) and also in the PC membrane W1 waveguide sketched in Fig. 1(d).

The photonic nanowire is a vertical GaAs cylinder with an embedded InAs QD placed on a substrate with an emission wavelength of $\sim 950 \mathrm{~nm}$. Let us consider the infinite photonic nanowire illustrated in Fig. 2(a). By choosing a proper nanowire radius $R_{n w}, \Gamma_{R a d}$ can be strongly suppressed as shown in Fig. 2(b), and the corresponding probability $\beta$ computed explicitly in Fig. 2(c) for an on-axis QD of emitting photons into the fundamental $\mathrm{HE}_{11}$ mode can be close to unity.

We notice that these designs do not employ a cavity and do not rely on resonant $\mathrm{cQED}$ effects to ensure a high $\beta$ factor, meaning that efficient coupling from the QD to the guided mode is obtained over a broad spectral range of several hundred nm as shown in Fig. 2(d). This means that spectral alignment between the emitter line and a narrow cavity line is not required, which represents a huge practical advantage in the fabrication.

While photons are efficiently funneled into the nanowire fundamental mode, half of the light will propagate towards the substrate due to the nanowire mirror symmetry. It is thus necessary to implement a bottom metal mirror to reflect this light back towards the top. Furthermore, the value of $R_{n w}$ which maximizes $\beta$ is only $\sim 0.13 \lambda$ leading to a narrow mode waist and a highly divergent far field emission pattern of the truncated nanowire. The top photonic nanowire designs thus incorporate a conical tapering strategy allowing for an adiabatic expansion of the fundamental mode and narrow output beam waist, thus ensuring a high $\gamma$. The original optically pumped photonic nanowire design [6] implements regular conical taper as shown in Fig. 1(b), and its first (a)

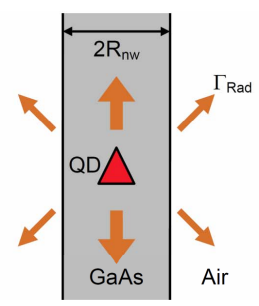

(c)

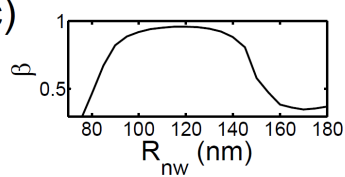

(b)

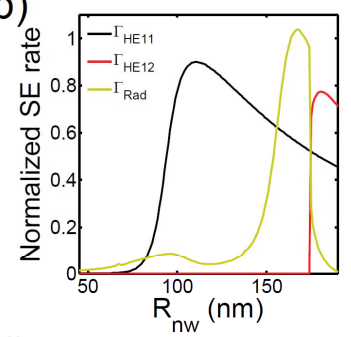

(d)

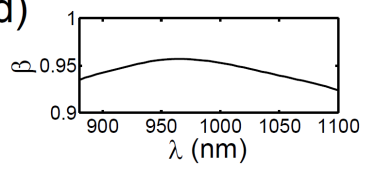

Fig. 2. (a) The infinite photonic nanowire with an embedded on-axis QD (red triangle). (b) Corresponding normalized spontaneous emission (SE) rates to the $\mathrm{HE}_{11}, \mathrm{HE}_{12}$ and the radiation modes computed for an in-plane QD dipole moment orientation and $\lambda=950 \mathrm{~nm}$. The $\beta$ factor as function of (c) nanowire radius $R_{n w}$ for $\lambda=950 \mathrm{~nm}$ and as function of (d) wavelength for $R_{n w}=120 \mathrm{~nm}$.

experimental demonstration featured an efficiency of 0.72. [7] However, for applications, electrical pumping is desired, and a novel photonic "trumpet" design based on an inverted conical tapering compatible with the implementation of a top metal contact as illustrated Fig. 1(c) has recently been proposed. [8] The trumpet features a strongly Gaussian far-field emission, and a first implementation of this strategy [9] has very recently lead to an ultra-bright single-photon source with a first-lens external efficiency of 0.75 and a predicted coupling to a Gaussian beam of 0.61 .

\section{REFERENCES}

[1] A. J. Shields, "Semiconductor quantum light sources," Nat. Photonics, vol. 1, pp. 215-223, 2007.

[2] W. L. Barnes, G. Björk, J. M. Gérard, P. Jonsson, J. A. E. Wasey, P. T. Worthing and V. Zwiller, "Solid-state single photon sources: light collection strategies," Eur. Phys. J. D., vol. 18, pp. 197-210, 2002.

[3] J. M. Gérard, "Solid-State Cavity-Quantum Electrodynamics with SelfAssembled Quantum Dots" in Single Quantum Dots, Topics Appl. Phys., vol. 90, P. Michler, Ed. Springer 2003, pp. 269-315.

[4] E. M. Purcell, "Spontaneous emission probabilities at radio frequencies," Phys. Rev., vol. 69, p. 681, 1946.

[5] O. Gazzano, S. Michaelis de Vasconcellos, C. Arnold, A. Nowak, E. Galopin, I. Sagnes, L. Lanco, A. Lemaître and P. Senellart, "Bright solid-state sources of indistinguishable single photons," Nature Comm., vol. 4, p. 1425, Feb. 2013.

[6] I. Friedler, C. Sauvan, J. P. Hugonin, P. Lalanne, J. Claudon and J. M. Gérard, "Solid-state single photon sources: the nanowire antenna," Opt. Express, vol. 17, pp. 2095-2110, February 2009

[7] J. Claudon, J. Bleuse, N. S. Malik, M. Bazin, P. Jaffrennou, N. Gregersen, C. Sauvan, P. Lalanne and J. M. Gérard, "A highly efficient single-photon source based on a quantum dot in a photonic nanowire," Nat. Photonics, vol. 4, pp. 174-177, 2010.

[8] N. Gregersen, T. R. Nielsen, J. Mørk, J. Claudon and J. M. Gérard, "Designs for high-efficiency electrically pumped photonic nanowire single-photon sources," Opt. Express, vol. 18, pp. 21204-21218, 2010.

[9] M. Munsch, N. S. Malik, J. Bleuse, E. Dupuy, A. Delga, J. M. Gérard, J. Claudon, N. Gregersen and J. Mørk, "Dielectric GaAs antenna ensuring an efficient broadband coupling between an InAs quantum dot and a Gaussian optical beam," Phys. Rev. Lett. in press. 